\title{
Ein ungedruckter Brief des Justus Jonas aus dem Jahre 1537.
}

\author{
Von Panl Vetter.
}

Im dritten Teile meiner Latherana ') babe ich anf die eigentumliche Rolle aufmerksam gemacht, die Jonas im Jahre 1537 gegenttber den Freiberger Superintendenten Jakob Schenck, einem der begabtesten unter den jungern schulern Lathers, spielte. Jonas' Verhalten gegen Schenck entspricht ganz und gar dem gegenuber Cordatus, als er im Jahre 1536 mit seiner Anklage gegen Melanchthon and Cruciger hervortrat ${ }^{2}$ ), und es ist wahrlich nicht sein Verdienst, daB Cordatus damals der Reformation nicht verloren ging. Auf das eigentumliche Verfahren gegen Witzel hat erst jüngst Kawerau in seinem ansgezeichneten Artikel in der Realenzyklopädie $^{y}$ ) wieder hingewiesen. Auch hier ist es Jonas, der einen verhängnisvollen Anteil an der Herbeifuhrung der Entfremdung zwischen Witze! und Wittenberg gehabt hat. Es läbt sich nicht leugnen, daß von den älteren Freanden und Mitarbeitern Lathers insbesondere er dessen Vertranen mibbraucht hat, um die einfluBreiche Sonderstellung in der neuen Kirche zo monopolisieren und aufstrebende, jilngere Kräfte zurilckzudrängen. In besonderem Maße ist dies bei Jakob Schenck ${ }^{4}$ ) geschehen, der 1536 nach Freiberg als Hofprediger an Herzog Heinrichs Hof gesandt worden war and sich bald durch sein Verhalten im Streite Lathers ${ }^{5}$ )

1) Neues Archiv f. Sächs. Geschichte XXX S. 76 ff.

2) Korstlin-Kawerau: M. Luther II $146 \mathrm{f}$.

ग) Theologische Realenzyklopädie XXI S. $401 \mathrm{ff}$., vgl. auch IX S. 346 a. Zeitschrift f. Kirchengeschichte XIII $287 \mathrm{ff}$.

4) Die Literatar über ihn: N. Archir f. Sächs. Gesch. XXX S. $76 \mathrm{f}$.

5) N. Archiv f. Sächs. Gesch. XXIX S. 82 ff. 
mit dem Herzoge sowie darch seine allzugroße Selbständigkeit bei den Wittenbergern miBliebig gemacht hatte. Als er Anfang 1537 sich dann an seinen Lehrer Jonas wandte, um gegenuber der wenig glucklichen Fassung der Visitationsartikel von 1528 an ihm einen Ruckhalt zo haben, da batte Jonas sich aalglatt der durchaus berechtigten Forderung zu entziehen gewaßt und einen Streit heraufbeschworen, in den schlieblich auch Melanchthon verwickelt warde. Im Verlaufe dieses Federkrieges hat Jonas, immer mit Luthers Autorität sich deckend, in wenig schöner Weise seine Stellong als Vizerektor der Universität benatzt, am Schencks Bruder Michael seine Predigtubangen in Wittenberg zo verbieten. Wieweit sein Anteil an der Entfernung Kargs ans seiner Stellung als Schloßprediger ${ }^{1}$ ) geht, ist nicht nachweisbar. Darch einen Machtsprach des Kurfursten wurde schlieBlich der Streit beendet und 1538 nach der Ernennung Schencks zam Hofprediger Johann Friedrichs ein wenigstens änßerlich leidliches Verhältnis zu den Wittenbergern wiederhergestellt. Im geheimen freilich wucherte der muhsam unterdruckte Groll weiter, und Luthers Tischreden ${ }^{2}$ ) geben uns ein anschauliches Bild von der Art und Weise, wie gegen Schenck gehetzt warde. Von besonderem Interesse ist das von Loesche ${ }^{8}$ ) mitgeteilte Tischgespräch, das Ende 1539 oder Anfang 1540 anzusetzen ist; denn Schenck ist noch unverheiratet und bewirbt sich um Bugenhagens Tochter. Die ersten beiden Sätze enthalten ein Urteil des Jonas uber Schencks Predigten, die hauptsächlich deshalb getadelt werden, weil er sich nicht darauf beschränkt, den Inhalt der loci Melanchthons oder einer Predigt Lathers wiederzugeben, sondern eigne Gedanken in seinen Kanzelreden entwickelt. Nach Luthers erklärenden Worten: „Herr Doktor, es ist ein alter Groll" spricht Jonas schadenfroh die Hoffnung aus, Bagenhagen werde Schenck die Hand der Tochter verweigern. Aus der Antwort Lathers erkennen wir, da $B$ die Verhetzangen zwar nicht ganz obne Erfulg gewesen sind, daß sein gerader Sinn sich aber doch anch dem vielangefeindeten Manne gegentuber ein selbständiges

1) N. Archir f. S. Gesch. XXX S. 93 u. 101.

2) Förstemann: Dr. Martin Luthers Tischreden III S. 372 a. a.

3) Loesche: Analecta Latherana et Melanchthoniana S. 56. 
Urteil vorbehält. Die Vermatang Joesches, daß die Lesart von $B 168$ in de magistro Georgio umzaändern sei, ist schwerlich richtig. Der Prozeß Kargs ${ }^{1}$ ), den Schenck durch seine Anzeige herbeigefthrt hatte, war längst beendet, und Karg hatte sich gerade dank seiner Gegnerschaft einer iberaus milden, entgegenkommenden Behandlang seitens der Wittenberger erfreuen durfen. Er befand sich damals, mit Lather versöhnt, in Öttingen in angenehmer Stellang. Eine Veranlassung fur Schenck, die längst abgetane, heikle Sache wieder hervorzuholen, ist nicht einzusehen. Die Notiz der Handschrift bedarf einer Verbesserung auch gar nicht. Am 1i. April 1539 war Herzog Georg von Sachsen gestorben. Es ist recht wohl denkbar, da $B$ Schenck seiner als eines Verfolgers des Evangeliums oder sonstwie in einer seiner Predigten gedachte ${ }^{2}$ ).

Von noch größerer Bedentung als die vereinzelten Spuren seines Hasses, die uns in den Tischreden entgegentreten, ist die Rechtfertignngsschrift, die Jonas am 18. Oktober 1537 an den Korfursten richtete. Von glubendem Hasse gegen den einstigen Schuler erfullt, wird sie za einer Anklage Schencks, dem freilich nur Undank und Hochmut vorgeworfen werden können. Sie enthält nicht die Verteidigung eines in seinen Rechten schwer gekränkten Mannes, sie ist das Schriftstuck eines geschickten Adrokaten, der dorch allerlei rhetorische Künste eine schlechte Sache zu verbrämen sucht.

1) Seidemann: Schenk S. $28 \mathrm{f}$. Wilke: Karg S. $17 \mathrm{ff}$.

2) Kroker: "Luthers Tischreden" ${ }^{\circ}$ zerlegt S. 84 ff. seiner Handschrift folgend die Stelle bei Loesche in $z$ wei Teile and bringt sie in umgekehrter Reihenfolge als selbutändige Tischreden. Hier haben Math. N und B 168 sicher das Richtige. Der erste Teil der Mathesiusschen Tischreden bei Kroker enthält übrigens nicht nar solche aus dem Jahre 1540, sondern auch aus früherer Zeit (so z. B. 29, 32, 71 n. ฉ.). Auch er bringt also nicht nar Selbstgehörtes, sondern es sind die Berichte anderer bereits in ihm mitverarbeitot. 


\section{Jonas an den Kurfürsten.}

Original. Die Unterschrift von Jonas eigner Hand. Weimarer Archiv.

Jhesus.

Durchlauchtigster, hochgeborner churfurst. E. Ch. G. seind mein vntherthenige. geborsame dienste zuuoran alzeit bereit. Gnedigster churfurst rnd her. Es het E. Ch. G. rath vnd cantzler, doctor Rruck, neulich d. doctori Martino, d. Philippo, d. Caspar Creatziger rnd mir eine schrieffit ${ }^{1}$ ) zagestellet, in welcher doctor Jacob Schencke kegen $\mathrm{E}$. $\mathrm{Cb}$. G. sich entschuldigen wil, warumb er bysanher of so genedigs begeren vnd schreiben, so E. Ch. G. aus sondern christlichen bedencken gethan, anher gegen Wittembergk za kommen. aus seinem eygen bedencken vnderlassen. So dan gemelter doctor, welcher sich aus grosser demath vnsern discipel nennet. in gemelten seinen schrifften mit mir auch ye etwas zu thuen baben will (wy ebr dan d. doctoris Martini selb nit verschonet vnd klaget vber inen in eynem wichtigen artickel, nemlich ehr habe seine, d. Jacobs aus Freiberg zugesehickte briffe ander lenthe lesen lassen), bit ich vatherthenigklich. domit doctor Jacobus im ersten jhar seines doctorats of sein schriefft nit ane antwort bleibe, mein vntherthenigen bericht gnedigklich zuuornemen.

Erstlich weys ich, gnedigster churfurst rnd her, nit. wie doctor Jacob Schenck seinem predigampt rnd grosser, schweren vocation, nemlich da ehr bernffen ist, wider den grewel des babstumbs das heilig enangelion in Meyssen vnther etlichen vielen boesen geystern vnd mensehen (so hoch wider vnser lere vorbittert sind) zu predigen, gedencket ein anfang zu macheu, ob das der richtige nehste wegk sol darzu sein, das ehr erst vns prediger vnd lectores zu Wittembergk. sonderlich d. philippum, der ime vil woltat ertzeiget hat. vor feynde achte vnd halte vnd aus freunden zu widersachen mit furgefasten, gesucbten matwillen mache, oder das ehr alhieher allein seinen bekanten freunden, forderern vnd praeceptoren trotzige stoltze brieffe ane noth schreibe. Des bin ich aber gewis, wan man in so hohen, wichtigen, gotlichen sachen sol etwas grosses ausrichten, das es wol if andere weyse mit allen propheten rnd aposteln zugangen, vnd das gottes geyst vnd christliche demut auch darzu gehoret. Vud Paulus sagt selbs, ebr achte der prediger nit

1) Der Brief ist verloren gegangen. Vgl. N. Archiv f. S. Gesch. XXX S. 95 . 
sehr, so furchtet sich Satan aucb nit seer vor jenen, die erstes anfangs of sich selbs so vil setzen, so prechtig hohe rede furen: dan I. Corinth. \& sagt er: Ich wil kurtzlich za 'uch kommen vnd erlernnen, nicht die wort der aufgeblasenen. sondern die krafft; dan gottes reich stehet nit in wortten. sonder in krafft. Do meyit $\mathrm{S}$. Paulus also, das werck solle den meyster loben, vnd wil der apostel, mit wortten sey es nit ausgericht, sondern wan gottes wort krefftig gehrt, wan greyst vnd gnad reichlich da ist, das viel hertzen von irtumb ind stricken Satane erlediget werden, wan vielen hertzen ind gewissen rechtschaffnen, bestendigen trost empfahen. wan ein feyn wacker glaube, freidickeyt vnd stercke des heyligen geists, recht gottesfurcht, recht christliche demuth vnd sanntfmut gegen jederman, rechte sorge vnd vleys, rotten Ind die hohen ergernis zu meyden, in predigern selbs ist, das sind zeichen eins treuen und rechtschaffen predigers. Was doctor Jacob mit seinen vnnötigen stoltz vnd trotz segen vns ind riel leuthen vor natz geschafft, wirt entlich offenbar werden.

Es hat doctor Jacohus vor vier jharen vngeuerlich von mir dorch den jungfrauschulmeyster alhie lassen bitten ein modum studendi et discendi in theologia, denen ich ime clar aufgezeichnet vod treuer, christlicher mainung mitgetailet. Dofur hat ehr mir offte gar freuntliche vleissige dancksagung gethaen, mit so grosser demuth sich erbotten ind so hoch of sich genbomen. der vnd ander vntherrichtung nymmer ewicklich zuuorgessen, das ich nit gemaint, das ehr in so kurtzer zeit die sprache gegen vns itzo in Meyssen vorandern solt, ond mag. gnedigster ber, mit warhait sagen, das doctor Jacobus wird machen, das ich alle vordechtig werd haben, welche so vf barfassermunche weyse mit $\mathbf{v b}$ rigen, erbitlichen, demutigen wortten sich hören lassen. Were nu dieselbige sein demath rechtschaffen gewesen, vnd wu ine vber das, das alle junge doctor (anch in weltsachen) ehr khune rnd trotzig sein dan ander, nit ein naturlich vbermuth anhinge, so het er es kunnen vbers hertze bringen vnd die Wittemberger (in ansehung, das dannoch ein junger doctor in solchen sachen raths wol bedarf) ime in angefangenem wercke helffen lassen. Aber der Titus bedarf keins Pauli, oder villeicht ist er ime selb in seinem synne ein Paulus rnd darf keyns Titi ader Timothei. Ich mainte, doctor Jacob, nachdem ehr erst vor wenig jaren angefangen in theologia zu studieren vnd ich seinen ersten anfang, mittel vnd ende wais, auch alle seiner knnst gewichte by of quintlein erkehent. solt noch zur zeit gerne das organum sein vnd die drometh (wie S. Hieronymus schreibt, das Titus Pauli organum gewesen) doctoris Martini vnd der Wittem- 
berger leer. So hat ehr sich in so kurtzer zeit so eben mit dem doctorpirret im spygel besehen rnd findet sich im ersten jar so reich von gaben, gelert, erfaren vnd gantz geschickt. das ebr drauen widder pfeiff noch harff zu sein gedenckt, sondern ehr wil der musicus, cytharedus, drometer wider Wittembergische gelerten vnd alles gar selb sein. Wan ehr nu, gnedigster herre, derselbig man gewis were. den er sich so zeitlich dencken lesset, so hetten gotfurchtige hertzen des weniger zu seufzen, vnd were die kyrche zu Meyssen (daran vns viel gelegen) dest besser bestellet, aber wieweyt es ime mangele. wirt in die zeit vod erfarung, der gemaine, starcke schulmeyster. leren. wan er nu von most des neuen doctorats nuchtern wird.

Dieses lang geschwetz wollen mir E. Ch. G. zo gut halten. Den es ist ye gewis war (wie auch diejenigen bekennen. so ime anbangen), das doctor Jacob ane alle not sich zu vns genotigt vnd vns mit trotz begegnet, vnd babe sorge, ime sei bange gewest. wie er sunst, ein solcher doctor. ein nhamen ind geracht erliff. wan er sich mit fluchs wider die von Wittembergk setrte. Were doctor Jacob warbaftig im hertzen so freuntlich vnd demutig gewesen, als er sich erstlich vnd sonderlich gegen mir stellet rnd munchisch geperdet, and wer berait, etwas arbeit. muhe oder fahr (wie er so auffigeblasen prechtig rumet) of sich zu nehemen, vnd het sich der mube nit lassen verdryssen, were, ehe rod zuuor ehr mit den brieffen vnd kartten umb den grossen doctornbamen and Teuerdanck mit vns Witternbergischen spylet, ja wer ehr bei tag rnd nacht gegen Wittemberg geraisset, hette freuntlich, bruderlich rath gesucht, oder hette vos zu im gefoddert, \%u underreden von artickeln, vom sacrament oder andern. wolte wir ime mit allem vleis. freuntlich bruderlich geratten haben; (w u es von noten gewesen) wolte wir ine gerne der kyrchen zu Freibergk vnd Meyssen zu gut mit schreiben, vaterrichten, predigen, leren treulich geholffen haben. Nun hat ehr vber alle vrsache. vber alles, das er vnsere gutwillig, bruderlich treue gemuth vnd hertz gegen ine wol albie erkhant, vber alle vnser freuntlich erbiten, auch vber alle bitten vod vermanen aus seinem heupt (dorynne villeicht viel rngelescht hyrn ist) diesem hohell wercke des ortts diesen anfang gemacht, welcher eyns auffgeblasen neulings furnhemen gleich siehet. herschet vnd waltet seins gefallens diesen tag ond hort nit auf, ane rath, ane gottesfurcht vnd demuth aus seinem aigen heupt prediger za setzen rad abzasetzen, rnd ist nun in den gedancken geratten. des babstumbs feuer sei dort durch inen, einen solchen man, rber die belfft geleschet, aber zu Wittembergk gebuere ime, als eynem neuen doctor. (obwol 
doctor Martinus auch ein wacker statwechter ist) zu wachen vod zu weheren. Mit denselbigen aber seinem vnbedechtigen furnhemen, welchs auch vielen verstendigen zu Freiberg vbel gefellt, die itzt schweygen, gibt ehr vrsach an alle not zu vielen reden, beschwerang vad ergernis, das seine zahorer das hertz von im abwenden, die widersacher eyn freude vnd wolgefallen haben. $O b$ das nun doctor Jacob den grossen schaden, welchen ehr dadurch perursacht. alles ror gott vorantwortten wyrdet, konnen E. Char. G. aus hohem christlichen verstand abnehmen vnd hat bei allen gotfurchtigen billichs bedencken.

Es kan, gnedigster churfarst vnd her, doctor Jacobus Init warheit vnd grunt nit sagen, das wir ime nit freuntlich vnd braderlich erstlich im anfang of sein schrieffte geantwort hetten; dan wiewol wir in E. Ch. G. vniversitet (da ein groß zal der studenten sind) vnd der kyrchen Wittembergk dermassen hie offt verhindert, das wir (ob doctor Jacobo nit allzeit nach der lenge antwort begegent) billich solten entschuldiget sein, so hab ich im doch erstlich gantz bruderlich, vortranlich vnd freuntlich widergeschrieben vnd gebeten, nit vngefallens zu tragen; ich achtet, alhie zu Wittembergk weren wol die artickel an besten zu conferiren, vnd alsdan wolt ich aigentlich eyntrechtiglich sampt doctori Martino ime mein bedencken zu erkennen geben; hab inen auch getrostet, wir wolten vor inen bethen, dort helffen predigen, wen er von vns begert. Vf solche freuntliche, vortrauliche schrieffte, bitten vad hoch erbieten hat der liebe junge doctor (der in seinem synne Paulus ist) vns armen Titos vod Timotheos von stund an verachtet vnd bald trotzige, hoffertige briffe geschriben, das d. Martinus vnd wir alle vns gesegnet, hoch verwundert, was ime doch gescheen were, das ehr yn eyner stunde vorgessen hette, wer hie an der elbe zu Wittembergk predigt; rnd hat mir mein tage keyn gelertter, der doch etliche namhaffig feind vnd freund za vns geschrieben, nye so plötzlich vnd vnuorarsacht mit hertickeit vnd trotz geschrieben. Derbalben haben wir (als auch schreiber) wie handwercksrecht rnd gewonheit geantwort vod inen noch zu vberflus vermanet, er wolte ane vrsach nit solch ergernis anrichten; dan alles, was er in schrifften fraget, könd er am allerbequemesten in beisein doctor Martini selbs mit vns albie reden. Was aber, gnedigster her, sein gethan schreiben an E. Ch. G. belanget, da er sich also entschnldiget, er konne derhalben auch vf E. Ch. G. gnedigs begeren gein Wittembergk nit komen, dan vor der widerkunfft m. g. h. hertzog Heynrichs von Wolckensteyn habe er nit konnen ron der kyrchen Freiberg ab sein, vnd es woll auch der gemain zu Freibergk schwer 
fallen, anch ferlich sein etc., konnen E. Ch. G. aus hobem christlichen verstandt, alle gottfurchtigen, denen die religionsachen mebr hertzlich vnd ernst ist dan doctori Jacobo. leichtlich abnebemen, was das fur ein kalt, kyndisch excnsation ist. Dan, gnedigster her, was solt da far ein schad vnd fhar vffstehen, ob das lant zu Meyssen ader Freibergk ein tagk oder zween doctorem Jacobum nicht hett? Ob er sich wol ans hohem vbermath Paulam achtet, warden doch viel fromer, christlicher leat, denen got durch seinen gaist das hertz geoffnet. die christliche leer za lesen vnd za horen. daramb nit Galatae werden. Wir wolten anch mitler zeit (wan ehr 80 viel bruderlichs gatten hertzen kegen vns haben kont), ehr aus Wittembergk doctor Craciger oder der prediger eynen hinschicken, domit dieweil der predigtstuel versorget mocht sein. Auch so ehr ein danckbar freantliche ader gat gemut trage gegen Wittembergk, so hette ehr d. Martinum vnd vns andern ken Torgau bescheiden konen oder ken Belgern, Leißnick, Colditz, wolten wir willig gerne komen sein. Do were aber villeicht durch den heyligen gaist vnd durch christlich, vetterlich einreden d. Martini dem nenen doctori Jacobo der wegk vorzennet vad vorbauet worden, das des jungen, nenen doctors roBleyn noch ebrn nit hette so schnel rnd hurttig lauffen konnen.

Ich wolt aber nicht gerne sein schult tragen, domit er sich alberait mit dem groben schertz fur got beladen hat; den was sinds vor rede? M. g. h., hertzog Heynrich war vfm Wolckenstein, item Freibergk kont meiner zwen tage ane schaden nit entperen, daramb mas ich, doctor Jacob. ergernis, scandala, geschrey, rede, klage vnd gerucht anrichten, die meiner zehen, ja hundert nit stillen kounen.

Es bet doctor Jacobus, gnedigster her, billich bedencken sollen, das ein solch jung man d. Martins rath alle stund wol in solcher gelegenheit bedarf, vnd das er vns danckbar zu sein schaldig ist, ond so bitter gemath aus eytelm amechtigen ${ }^{1}$ ) ehrgeitz nit fassen, als bet ime die Wittemberger nit ein doctorpirret, sondern ein gluend eyssenhat vffgesetzt. Paulas selb, der ein wenig ein hoher doctor gewesen vnd andern malfasier gschanckt, dan doctor Schenck ${ }^{2}$ ), setzt warlich so hohe sachen nit of sich allain trotzlich oder stoltzlich, bittet vnd flehet Titum rnd Timotheum, das sie wollen helffen erbeyten, weren ond wachen wider den Satanam, belffen trosten, vermanen, tragen vnd leiden, bethen, gen hymmel ruffen, ane rnterlas anhalten, vod of die weyse gehets an pflantzung des enangelii. Homath that nie keyn

1) = schwach.

2) Eine Anspielung auf Schencks Doktorschmans; vgl. Seidemann: Schenk S. 11 u. 98. sowie Drews: Disputationen Dr. M. Lathers S. 99. 
guth. Als er, doctor Schenck, ein man ist, rnd als starcken wein in gerstlichen sachen er noch schencket, het er sich dem exempel nach solcher demath nit schemen dorffen. solt sich aber viel mehr gegen E. Chur. G. furnemlich, als den bochuorstendigen, christlichen churfursten, allen gelerthen vnd vorstendigen ewiglich schemen des plotzlichen rnd vnuorsehelichen ${ }^{1}$ ) vbermuths kegen Wittembergk. Wir mugen wol mit Panlo sagen: Was haben wir in doch gethaen? Alles gatts haben wir ime gethan. $\mathrm{Na}$ das wol er vns vorzeyhen, ehr hette ye (so rechte gottesfurcht in ime were) bedencken sollen vad ernstlich za hertzen furen, das sein abwesen zwen oder dreyer tage von Freibergk viel ein geringer nachteyl oder schade were, dan mit solchen zuuotigen(!) vnd trotzigen schreiben ane not (so man ime freontlich vnderredung im anfang angebothen) in diesen verlichen zeyten zu abschrecken vieler gotfurchtigen, beschwerung, ergernis vnd so grossen scandalis vrsach geben. Aber das hat ehr anfengklich gesucht, das er ehre erliffe rnd des neuen doctors name bekant werde; es kau aber kain seugekindt oder knab kein koriber sein, mus doch erharren, das er erwechst; also mus d. Jacobus harren, das in got aufwachsen lasse, zu seiner lenge wirt er kein fingerbreit selb setzen konnen.

Wo er nu vber alle vnser erbiten vnd e. ch. g. gnedigs begeren anherznkomen sich beschweret, anch vit leiden mag, das wir alle, im zu ebren, im ersten jar seins doctorats kegen Belgern enkegen ziehen, gedenckt die sache dahin zu erbeyten, das d. Jacob Schencke in buchladen kome, so werden wir dermassen schreiben, das wir fur got vnd alle welt furantwortten wollen; dan ich far meyn person scheu des widersachers nit.

Das aber doctor Jacobus weitter in der schrifft an E. Ch. G. anzeigt von seiner person mit diesen wortten: „Den es sol mir, d. Jacobo, zn Wittemberg schult gegeben werden, wie ich es alles woll allein sein vnd ane ir halff ausrichten, hab auch das babstumb ane irn rath, wissen vnd mithnlff abgethan, welchs nun auch zu Freibergk statruchtig “ (diese sint d. Jacobi wort). Aus diesen wortten merckt man wol, das d. Jacob Schencke sich vor ein man achtet, wiewol er gar noch vnerwachssen zum ernste vnd rechten ritterspiel, ron dem leuth zu Parys, Löuen, Wittembergk vnd andern berumpten schalen vielen vergeblich rede treiben.

Vnd ich hab einmal von d. doct. Martino ein apologam gehort, das sechs starcke reyssige hengste haben anf ein zeit ein lastwagen im sommer in durrem sande gezogen,

1) oder vnnorstehelichen. 
ist ein groB stanb auffgangen, das man wider farman ader pfert wol bat sehen konnen. Do ist ein nasse flige oben gesessen an den wagenbeumen vod hat sich vordechtig gehalten, als hab sie den stanb erwecket, vnd hat daruber geschwitzt vor angst. Also mag villeicht in dem stuck der doctor sorge tragen der dinge, der niemands inen vordenckt: den er hat sich viel hoher verdechtig von schaden zo thuen. dan jemands dencket, das ebr willen habe zu than oder zu wircken mit allen krefften vermoge.

Weitter, gnedigster her, das der doctor Jacob die sachen seins bruders Michel Schencken E. Ch. G. auch furbringet rnd in derselbigen supplication mit vnsers hergots rod der religion sachen allein ein blat, aber mit des bruders sachen sechs gantz bletter zubrenget rod es vmb den bruder gar lesset zu thun sein. welchen ehr als an vaters vnd preceptors stadt ime hochlich vud ewigk zu eheren furnimbt ${ }^{1}$ ), das las ich $\mathbf{E}$. Ch. G. vnd allen vorstendigen zu bedencken stehen vod wil mit d. Jacobo seins braders halben, ader ob er eyner besoldung eynes predigers zu Freiberg oder anderu ortten wirdig sey oder nicht, nit groß fechten. er mag khinder, schmiedknecht oder schichtmeyster zu predigern aufstellen, weys ers vor got zuuorantwortten. Seiner oder mein brader mit iren solden vnd prebenden bleiben, wa sie wollen, so sollen billigh die sachen der ersten taffel decaloge. gotts wort, gotts dinst, vermeydung ergernis belangend furgehen.

Doctor Martinus vnd ich habe seinem bruder Michel Schencken (welcher ein schichtmeyster im Tall $^{2}$ ) gewesen) diesen beuehel geben, das er zwei jar studieren solte, darnach sich examiniren lassen; sei er alsdan zu predigen geschickt, solle er zum predigambt gefordet vnd gebraucht werden. Vnd was ich vor mein person als vicerector oder sunst mit Michel Schencken schichtmeyster geredt oder gehandelt oder mit wissen d. Martini reden lassen, bin ich erbutig, mundtlich, wan ich erfordert werde, vntertheniglicheu. claren bericht za thuen, wie dan d. doctor Martinus E. Ch. (;. allbereit zum tail gethan ${ }^{8}$ ).

Das aber doctor Jacob im selbigen ausgebreyteten schreiben vom brader (das auch ime seer anligt) vnter andern diese wort braucht: „Der her probst hat mein bruder etwa lassen zu sich foddern vad in einem schwinden, grimmigen, vnheymlichen zorn bedrauet, er wolle mich darzu halten, das ich weitter an inen vnd an Philippam nit schreibe,

1) Vgl. hierzu Burkhardt: Lathers Briefwechsel S. 284.

2) Joachimstal vgl. $\Lambda$. Archiv für S. Gesch. XXX S. $90 \mathrm{f}$.

3) Iu Lochan Anfang (Oktober. N. Archir f. S. Gesch. XXX S. 99. 
sunst wurde es vos beyden rbel gehen." item als sol ich gesagt haben, ich konne der sacben ane zorn nit gedencken. item das ich mir hab sollen gefallen lassen, das Micbel Schencke im dorf Brate ${ }^{1}$ ) gepredigt. so er zunor von niemands examinirt, dieses alles hat d. Jacob E. Ch. G. so neydisch vnd mit so gantz vnguttigen wortten furgetragen, das ich mich verwunder, das er in brieffen an vns so offte sein todt vmb Christi vnd des enangelii anbentet vnd vmb seiner aigennutzigen haus- oder kluchensachen willen mit so geschwinden zorn, neidt vnd haß sich mercken lesset.

Es ist d. Jacobo an der religionsachen viel gelegen vnd ist im doch am schichtmeyster, dem brader, auch etwas gelegen; das er aber vormeldet, sein bruder hab im dorf Brata drei predigten nach eynander gethan vnd domit seinem. Jacobi, exempel gefolget, der die erste wochen seines anhebens funf predigten gethan, domit er das erste schrecken vberwunde, hat sein bruder beymlich ane $d$. Martini oder der visitator beuehel ime rorgenhommen zn predigen, darumb ists im auch vnthersaget. So ists auch nit wunder, das d. Jacob-viel predigten kurtz vffeynander thuen kan; dan er hat sich zu Freibergk rernhemmen lassen, wan er das buch ansehe, so hab er ein predig gefasset. Warlich der ramb ist nicht niederick, wo er anders nit zo hoch ist. Ferner das d. Jacob aus dem milden, guttigen gemut, so er gegen seinem forderer vnd preceptorn tregt, angezeigt. ich sol geredt haben, es sei den von Wittembergk ein schandt, das sie ein schichtmeyster leren solle, sind mir abermal mein wort warlich vnfrentlicher weyse vmbgekhert. Icb halt aber dannoch, E. Ch. G. werden doctor Martinum lieber bie vor eyn prediger wyssen, dan ein fischer oder schichtmeister, es weren dan fischer wie S. Peter.

Das doctor Jacob auch claget, sein bruder sei wider im lateiu noch christlicher lere gefragt worden, ist ehr die zeit za kainem examen bescheiden, vnd bin erbutig vrsach anzuzeigen, waramb man demselbigen schichtmeyster obangezeigten beschait (predigens sich zu enthalten) geben hat. $\mathrm{Ob}$ aber $d$. Jacobus seinen bruder aus geneigtem willen gelerter grumet ${ }^{2}$ ) mit vielen prechtigen wortten dan sich selbs in gotlichen sachen, lassen wir ein seinem werd; wan wir nit offentlich mirakel sehen vnd feurige zungen, wie am pfingstag, so bleiben wir bei der heiligen schrifft vnd lere doctor Martini vnd halten in E. Ch. G. schule strack schulrecht, lassen keynen fischer noch schichtmeyster zum predigambt, wir horen erst, ob er etwas rom latein, grammatiken

1) Pratau bei Wittenberg.

$\left.{ }^{2}\right)=$ gerühmet. 
Ind biblien kau, vnd that darzu nichts, ob Cicero durch die kunst nit in hochsten himmel erhoben ist. Dan wan auch kunste vnd latein solte gantz $\mathrm{vmb}$ der schichtmeister vnd fischer willen veracht werden, so warde es regimenten. guten, nutzlichen gesetzen, legibus vnd iuribus rnd kyrchensachen nit geringen schaden thuen, vnd ein junger doctor, der noch nagelneu ist, solt billich dem hantwerck rnd allen ebrlichen kunsten $z a$ eheren anders ron sachen schreiben. sunderlich E. Ch. G., welche itzunt in dentzscher, welscher nation, vielen landen rnd kunigreichen berambt sind, das sie eraditionem veram $z$ p pllantzen vnd bys of die nachkommen zu forderang der religion rnd waren gottesdienst $\mathrm{zu}$ erhalten, viel vnd hochsten vleis furwenden.

Dan es hebet auch nit hoch in hymmel, wan eyner gleich, Ciceroni vngleich, vngelert, rngeubt, allen schulen rnd knnsten (dy arbeit vad vleis erfodern) feind ist; vnd von doctor Jacob ists mir ein seltzam rhom, das eyn schichtmaister (weyl er sein bruder ist) so hohen rumb in spiritualibus bei ime hat, rnd das doctor Martinus ond alle Wittemberger so sperlich gelobt werden. Was doctor Jacobus seins gefallens vom hohen rectorampt aber ans seiner gewonlichen danckbarkeit kegen vns houisch meldet, will ich vbergehen: dan er that mir vnrecht. Dan was ich mit seinem bruder vnd $m$. Jeorg Karck seinthalben etlich mal geredt babe. bin ich vatherthenigklich erbutig, gutten goungsam bericht zu thuen.

Entlich so gemelter doctor ein gelerther vnd gewisser theologus durch nit stilschweigen rnd $\mathrm{ryl}$ schreiben gedenckt zu werden (welchs dannoch ehr nit vngereymbt bedacht hat), wolten wir ime anch wunschen, das ehr E. Ch. G. gnediger, schrifftlicher vorynnerang ${ }^{1}$ ) volgete vnd etwas ans den veteribus: Augustino, Hieronymo vnd andern kyrchenhystorien tuglichs wider das babstumb zasamenbrecht vod sein vnerschrockene tapfere manheyt er erst an feinden beweysete.

Wie dem nu allen, g. ch. vnd her. so haben E. Ch. G. (wie ich ans E. Ch. G. gnedige schriefft an doctor Jacoben vormarekt) diese sachen vil vleyssiger (dan ich itzunt daruon anzaigen kan) auch gantz furstlich vnd christlich bedacht vnd gemelten doctor $z a$ foderlicher mundtlicher vnterredung vod vormeidung alles gezeugnis (!) vnd vorbitterung (wie iuch S. Paulas sein christen warnung that) gnedigklich forbescheiden. Nachdem auch E. Ch. G. ime clar mit dentlichen vnd geigen rns Wittemberger gantz gnedigen wortten in schriefften anzeygen, das ehr, mit nicht stilschweygen

1) N. Archiv f. S. Gesch. XXX S. 96. 
eyn geterther vnd gewisser theologus $\mathrm{zu}$ werdeu. bequemer seins schreibens widder vaser adversarien brauche muge dan wider vns, sint wir $\mathrm{E}$. Ch. G. vnterthanig dancksagnng schuldig. Vnde zu beschlus anch fur mein person bit ich auch gantz vnderthenigklich. E. Ch. G. wollen gnedigklich bedencken, das vnser hergot vast vor zweintzigk jharen (da doctor Jacob noch etwas junger geweben) des seligk liecht des heyligen enangelii durch doctor Martinum hat erforbracht. ind das von dem ersten reichstag an zu Augsparg zun zeitten Maximiliani vnd ron dem ersten Wurmischen reichstag an bys of dieses XXXVII. jhar sint d. Philippus. d. Ponmeranus vnd ich doctoris Martini discipel vnd junger gewesen, mitlerzeit neben doctor Cratziger vnd andern allen vleis gethan, in den schulen vnd kyrchen vielen za gat reyne christliche lehr $\mathrm{zn}$ erhalten; wollen anch got noch veissig bitten, das er vns gnade vorlei, bei dem heyligen cuangelio vnd E. Ch. G. confession, auch bey E. Ch. G. leib vnd leben vnd alles zuzusetzen, damit die reyne christliche lere, rechtschaffiner vnd gewisser trost der selen vnd gewissen ausgebraitet vnd erhalten werde. Vnd bitten auch also: E. Ch. G. wollen gnedigklich rorschafen vnd daran sein, das, wan wir hie zu Wittembergk mit predigen, lesen, schreiben, vieler mube, allerlei kirchen- vnd schularbeit E. Ch. G. gehorsamlich dienen, auch andern kirchen, landen vnd steten zu gut, so viel vns got gnad geben, burden tragen, vns in vnserm alder vorthin nit ein itzlicher solcher doctor, vnser discipel, vberrumpele vnd seins gefallens mit hartten schrifften vouorarsacht belade. Dan wiewol Dauid im psalter von sich selber redt rnd von andern gotfurch. tigen, so geistliche weybheit reichlich haben, saget: Super senes intellexi. so seint doch alle junge doctor nit Dauid. Wan es nutz und gut sein solt vnd wir nit lieber die kirche Freibergk rnd gantz Meyssen vfs best erbauet, dan im anfang zertrennet sehen, were dem jungen doctor mit schrifften wol stercker vnd gerusteter za begegnen, deu ehr noch geritten ist. E. Ch. G. werden an allen zweifel, ergernis zunorhatten, gnedigk einsehen haben; dan vornemlich kan ich doctor Jacobi halben des herrn doctor Martini erkentnis allzeit wol leiden, vnd so es anch not were, konnen wir Wittemberger (wie vns doctor Jacob etwa als fremde Indianer oder Hispanier, ime gar vnbekant nennet) auch leiden, das E. Ch. G. als der christlich, hochloblich churfarst, alle farsten rnd stende dem euangelio verwant, alle gelerthen: Brentius, Urbanus Rhegius, Osiander et cetera, ader auch aus fremden andern nationen (so der religion mit vns nicht vneynigk) diese sache anhören vnd orttern. Beuele mich hiemit E. Ch. G., bin derselbigen in aller vnderthenigkeit vnd gehorsam allzeit 
zu dienen willigk rnd geflissen. Datum Dornstagk nach Burckardi ${ }^{1}$ ) anno dni 1537.

E. Ch. G.

$$
\begin{aligned}
& \text { vntertaniger williger diner } \\
& \text { Justus Jonas doctor } \\
& \text { p. Vittembergk. } 8 .
\end{aligned}
$$

1) 18. Oktober. 